\title{
Dificultades en los métodos de estudio de exposiciones ambientales y defectos del tubo neural
}

Víctor Hugo Borja-Aburto, M.C., M.S.P., M. en C., Ph. D., ${ }^{(1)}$ Ó scar Bermúdez-C astro, M.C., ${ }^{(2)}$ Marina Lacasaña-N avarro L.F., M. en C., Ph.D., (3) Pablo Kuri, M.C., M. en C., (4)

Patricia Bustamante-Montes, M.C., M.S.P., M. en C., ${ }^{(5)}$ Víctor Torres-M eza, M.C. ${ }^{(1)}$

\section{Borja-A burto VH, Bermúdez-Castro 0 , Lacasaña - Navarro M, Kuri P, \\ Bustamante-Montes P, Torres-Meza V. Dificultades en los métodos de estudio de exposiciones ambientales y defectos del tubo neural. Salud Publica Mex 1999;41 supl 2:S124-S131.}

\section{Resumen}

Objetivo. D iscutir las actitudes en la evaluación de las exposiciones ambientales como factores de riesgo para defectos de riesgo del tubo neural, al tiempo que se presentan los principales factores estudiados hasta la fecha. Resultados. Las exposiciones ambientales se citan muy a menudo como causa de malformaciones congénitas; sin embargo, ha sido difícil establecer esta asociación en los estudios de poblaciones humanas, debido a problemas en su diseño y conducción. Lo anterior es particularmente marcado en el caso del estudio de los defectos del cierre del tubo neural (DTN), que es una de las principales malformaciones y que incluye anencefalia, espina bífida y encefalocele, y su asociación con exposiciones ambientales. Las dificultades en los métodos surgen de: a) la medida de frecuencia para realizar comparaciones espacio-tempo rales; b) la clasificación y heterogeneidad de las malformaciones; c) la consideración de los factores relacionados con la madre, el padre y el producto, de manera conjunta,y d) la evaluación de las exposiciones ambientales. Conclusiones. Hipotéticamente las exposiciones ambientales tanto del padre como de la madre pueden producir daño genético antes y/o después de la concepción por la acción directa sobre el embrión o sobre el complejo fetoplacentario, de tal manera que en la evaluación de exposiciones ambientales: a) deben tomarse

\author{
Borja-A burto VH, Bermúdez-Castro 0 , \\ Lacasaña-Navarro $M$, Kuri $P$, \\ Bustamante-Montes P, Torres-Meza V. \\ Methodological challenges \\ to assess environmental exposures related \\ to neural tube defects. \\ Salud Publica Mex 1999;41 suppl 2:S124-S131.
}

\begin{abstract}
A bstract
Objective. To discuss the attitudes in the assessment of environmental exposures as risk factors associated with neural tube defects, and to present the main risk factors studied to date. Results. Environmental exposures have been suggested to have a roll in the genesis of birth defects. However, studies conducted in human populations have found difficulties in the design and conduction to show such an association for neural tube defects (anencephaly, espina bifida and encefalocele) because of problems raised from:a) the frequency measures used to compare time trends and communities, b) the classification of heterogeneous malformations, c) the inclusion of maternal, paternal and fetal factors as an integrated process and, d) the assessment of environmental exposures. Conclusions. Hypothetically both maternal and paternal environmental exposures can produce damage before and after conception by direct action on the embryo and the fetus-placenta complex. Therefore, in the assessment of environmental exposures we need to take into account: a) both paternal and maternal exposures; b) the critical exposure period, three months before conception for paternal exposures and one month around the conceptional period for maternal exposures; c) quantitatively evaluate environmental exposures when possible, avoiding a dichotomous classification; d) the use of biologi-
\end{abstract}

(1) Centro N acional de Salud A mbiental, Dirección General de Salud A mbiental, Secretaría de Salud (SSA), México.

(2) Centro de Investigación y Estudios Avanzados, Instituto Politécnico N acional, México.

(3) Centro de Investigación en Salud Poblacional, Instituto N acional de Salud Pública, México.

(4) Dirección General de Epidemiología, SSA, México,

(5) Universidad Autónoma del Estado de México, Toluca, Estado de México, México.

Fecha de recibido: 13 de mayo de 1998 - Fecha de aprobado: 5 de marzo de 1999

Solicitud de sobretiros: Dr.Víctor Hugo Borja Aburto. Centro N acional de Salud A mbiental, Dirección General de Salud Ambiental, Secretaría de Salud. A partado Postal 37-473,06696 México, D.F., México.

Correo electrónico: vborja@ toluca.podernet.com.mx 
en cuenta las exposiciones maternas y paternas; b) debe considerarse el periodo crítico de exposición, esto es, tres meses anteriores a la concepción para el padre y un mes alrededor de la concepción para la madre; c) en la medida de lo posible, la evaluación de la exposición deberá ser cuantitativa, evitando clasificar a los grupos únicamente como expuestos y no expuestos, y d) es recomendable emplear marcadores biológicos de exposición siempre que sea posible, así como utilizar marcadores biológicos que permitan clasificar a la población en grupos con distinta susceptibilidad genética.

Palabras clave: defectos del tubo neural;exposición a riesgos ambientales cal markers of exposure is highly recommended as well as markers of genetic susceptibility.

Key words: neural tube defects; environmental exposure
$\mathrm{L}$ a disminución de las enfermedades infecciosas en la infancia ha ocasionado que las malformaciones congénitas ocupen cada día un lugar más importante como causas de enfermedad y muerte en la infancia en la mayoría de los países desarrollados y en algunos en desarrollo. ${ }^{1}$ En los Estados Unidos de América (EUA) las malformaciones congénitas son la principal causa de mortalidad infantil: en $1988^{2,3}$ contribuyeron con cerca del $21 \%$ del total de muertes infantiles. Se estima que cada año, en los EUA, nacen entre 100000 y 150000 niños con algún defecto mayor. ${ }^{4}$ En México, uno de cada 50 recién nacidos vivos, y uno de cada nueve nacidos muertos, presentan una o más malformaciones mayores o menores ${ }^{5}$. A pesar de que las malformaciones congénitas ocurren con relativemente poca frecuencia, constituyen un problema de salud pública. ${ }^{1}$

Uno de los problemas más preocupantes relacionados con las malformaciones congénitas es que se sabe muy poco acerca de su etiología. Se estima que $60 \%$ de todas las malformaciones tienen una causa conocida; dentro de ese grupo, $7 \%$ corresponden a defectos en un solo gen, mientras que los defectos de etiología multifactorial comprenden al 20\%. Esta última categoría es el resultado de la interacción de factores genéticos y ambientales. ${ }^{6}$ Hace no más de 30 años la opinión científica atribuía las anormalidades congénitas a causas puramente genéticas. El descubrimiento de agentes teratógenos humanos como el virus de la rubéola, la talidomida y el mercurio orgánico, entre otros; así como las experiencias de investigación en animales, han modificado esa opinión, de tal manera que actualmente no se puede subestimar la importancia de los factores ambientales en la génesis de las malformaciones congénitas.?

De particular importancia resultan las exposiciones ocupacionales de hombres y mujeres a una can- tidad cada vez mayor de sustancias químicas que se utilizan en la industria y la agricultura. Las mujeres se incorporan, de manera creciente, a la fuerza laboral; un gran número de ellas lo hacen en la etapa reproductiva de la vida, lo cual posiblemente ocasione una mayor cantidad de eventos reproductivos en riesgo.

Entre las principales malformaciones, por su frecuencia e impacto en las familias que los sufren, se encuentran los defectos del cierre del tubo neural (DTN) que incluyen anencefalia, espina bífida y encefalocele. ${ }^{8}$ Normalmente el cierre del tubo neural es un proceso continuo que empieza en la región cervical y progresa rostral y caudalmente; ${ }^{9}$ sin embargo, ciertos factores genéticos y ambientales retardan o interfieren con este proceso de cierre normal. Las exposiciones ambientales tienen efectos potencialmente teratógenos a través de distintos mecanismos, como son las alteraciones en la diferenciación celular y en la migración celular, la muerte celular excesiva o, en algunos casos, inadecuada, y las alteraciones en la comunicación intercelular y/o alteraciones del metabolismo. ${ }^{10}$ En el caso de muchas exposiciones ambientales que se sospecha están relacionadas con defectos en el cierre del tubo neural, estos mecanismos no se han establecido aún y, por otra parte, no cubren los criterios de causalidad para teratogénesis que han propuesto Kline, Stein y Susser. ${ }^{11}$

El mayor logro en la investigación sobre los factores que intervienen en los DTN es la demostración de su prevención a través del suplemento con ácido fólico y multivitamínicos en la etapa periconcepcional. ${ }^{12,13}$

La sospecha de que las exposiciones ambientales causan malformaciones congénitas es tal, que se les ha propuesto como eventos centinela para detectar peligros ambientales y ocupacionales, sin que se haya demostrado hasta el momento la utilidad y la etiología 
de los mismos, sobre todo debido a dificultades en los métodos de estudio en poblaciones humanas. Destacan las dificultades en: a) la definición de la medida de frecuencia para hacer comparaciones espacio-temporales; b) la clasificación y heterogeneidad de las malformaciones; c) la consideración de los factores relacionados con la madre, el padre y el producto de manera conjunta; y, d) la evaluación del las exposiciones ambientales.

\section{Comparaciones geográficas y temporales de los DTN}

La sospecha inicial de que los factores ambientales contribuyen en la etiología de las malformaciones congénitas proviene de estudios ecológicos de comparaciones temporales y espaciales de la frecuencia de los DTN entre poblaciones. La incidencia de estas malformaciones tiene variaciones geográficas; además, en familias de inmigrantes la incidencia parece ajustarse hacia el nivel de la nueva población a la cual se integran. ${ }^{14}$ Estas variaciones se hacen evidentes en brotes o epidemias cortas, tales como conglomerados en lugar y tiempo, ${ }^{15}$ con tendencias descendentes en algunos países. En los EUA, las tasas disminuyeron de $20 \times 10000$ nacimientos en la década de los setenta, a $8 \times 10000$ nacimientos en los ochenta. Asimismo, la literatura indica que la prevalencia de DTN es más común entre mexicanos que en caucásicos en Norteamérica ${ }^{15}$ y que hay un gradiente de riesgo con prevalencias más altas en México, más bajas entre caucásicos de los EUA, e intermedia entre población descendiente de mexicanos en los EUA. La reducción de los DTN en algunos países podría deberse a la terminación electiva de los embarazos afectados que fueron detectados por el tamizaje y diagnóstico prenatal. ${ }^{16,17}$

Sin embargo, en las comparaciones geográficas $y$, sobre todo entre países, es necesario tomar en cuenta algunas dificultades de la comparación misma, debidas a criterios distintos en la estimación de su frecuencia, por diferencias en la definición y clasificación de los casos. ${ }^{16,18}$ Así por ejemplo, en algunos informes se ha incluido, erróneamente, a la hidrocefalia en este grupo. Asimismo pueden presentarse diferencias en los métodos de búsqueda y registro de los casos, por diferencias en los sistemas de vigilancia, así como en la definición del denominador para el cálculo de la prevalencia. No siempre hay una diferencia clara entre la edad gestacional para considerar los abortos y las muertes fetales y cuáles de éstos son incluidos en el denominador. Por otro lado, en estas comparaciones es necesario tomar en cuenta los cambios seculares observados en varios países. Si estos factores no se unifican entre regiones o países, las comparaciones geográficas difícilmente pueden servir como generadoras de hipótesis..$^{16,18}$

En México sigue siendo difícil estimar con precisión la frecuencia de DTN; a pesar de que los registros han mejorado de manera sistemática, aún no se tiene una cobertura universal. El Sistema de Vigilancia Epidemiológica de los Defectos del Tubo Neural se estableció a partir de la necesidad de información que había en algunas ciudades prioritarias de la frontera norte y otras más de la República mexicana, con fines de comparación. ${ }^{19}$ Para las ciudades prioritarias, en 1995 se observaron prevalencias relativamente elevadas, como en Matamoros y Reynosa, con 12.8 y 7.5 por cada 10000 nacidos vivos registrados, respectivamente, mientras que para las ciudades de comparación, Puebla, con prevalencia de 37.9, ocupó el primer lugar; le siguieron Tlaxcala y Querétaro con 14.3 y 14.2, respectivamente, por cada 10000 nacidos vivos registrados. ${ }^{20}$ Actualmente se cuenta con registros completos de las malformaciones que llevan a la muerte, gracias a los certificados de defunción; sin embargo, éste no es el caso para las malformaciones que son compatibles con la vida y menos evidentes que las anteriores.

\section{Clasificación y heterogeneidad de los DTN}

Como ya se ha mencionado, los DTN incluyen la anencefalia, la espina bífida y el encefalocele. Algunos investigadores han tratado estas entidades como un grupo homogéneo; sin embargo, desde hace algún tiempo otros autores han llamado la atención sobre la heterogeneidad de estos defectos. Estas tres patologías son anatómicamente distintas y también podrían tener etiologías distintas. ${ }^{21-23}$ Esas diferencias en la etiología estarían fundamentadas en los incrementos diferenciales en anencefalia o espina bífida, principalmente anencefalia, y no en los dos, paralelamente, en los brotes de DTN. ${ }^{7}$ La exposición a ácido valproico se ha asociado más a espina bífida que a anencefalia. ${ }^{24}$

Por otra parte, los investigadores no han empleado los mismos métodos de confirmación de casos, de tal manera que algunos recurrieron a los informes médicos, mientras que otros confiaron en el informe de la madre. En Colombia, Restrepo y colaboradores ${ }^{25}$ notificaron una gran proporción de falsos positivos que habían informado los padres. 


\section{Factores relacionados con la madre, el padre y el producto}

En el estudio de la etiología de las malformaciones congénitas es necesario considerar la intervención de los tres participantes en el proceso reproductivo: el padre, la madre y el producto (embrión, feto, niño). Las exposiciones ambientales tanto del padre como de la madre pueden producir daño genético, o mutagénesis, antes y/o después de la concepción por la acción directa sobre el embrión o el complejo fetoplacentario (teratogénesis). ${ }^{26}$ La justificación para el estudio de una posible relación entre la exposición laboral del padre y el subsecuente desarrollo de una malformación congénita en sus descendientes, se basa en que las exposiciones a riesgos potenciales pueden afectar las células germinales antes de la concepción, o bien las células somáticas embrionarias después de la concepción. ${ }^{3}$ Las malformaciones pueden producirse a través de daño genético antes de la concepción y/o por acción directa sobre el embrión o feto; ambos mecanismos pueden operar a través de la exposición masculina o femenina y en diferentes momentos en relación con la concepción y el embarazo. ${ }^{26}$ Las exposiciones paternas pueden producir, directamente, mutación de las células germinales, lo cual se expresaría en malformaciones en generaciones subsecuentes; además, la presencia de toxinas en los fluidos seminales y la contaminación de ropa de trabajo que se lleva a casa pueden causar exposición secundaria de la madre. ${ }^{9}$

Si bien las exposiciones previas a la concepción son importantes, el mayor riesgo de teratogénesis por exposición de la madre se relaciona, generalmente, con exposiciones durante la fase de organogénesis. $\mathrm{Mu}-$ chas sustancias químicas pueden atravesar la placenta y llegar al embrión durante los periodos de mayor diferenciación. Por otro lado, las exposiciones maternas en periodos previos al embarazo pueden acumularse en los tejidos corporales y liberarse al torrente sanguíneo durante la gestación, produciendo así un daño retardado en la exposición. Otro posible mecanismo, poco estudiado, podría ser la afectación de las células germinales por exposición de la madre durante su época fetal y la manifestación posterior en la siguiente generación. ${ }^{11}$

\section{Evaluación de exposiciones ambientales}

En el estudio de DTN, la evaluación cuantitativa y temporal de la exposición a contaminantes ambientales es un asunto clave. La mayor parte de los estudios se han basado en la ocupación de los padres asentada en los registros rutinarios; en otras investigaciones se han empleado cuestionarios, y en otras más se ha recurrido a la evaluación de probabilidades de exposición asignadas por higienistas industriales o a través de la aplicación de matrices de exposición. Sin embargo, no se han investigado los componentes activos específicos, como el caso de los plaguicidas, de tal manera que en ocasiones la ocupación se ha relacionado más con el nivel socioeconómico que con exposiciones ambientales y ocupacionales específicas.

A menudo la evaluación de la exposición se realizó de manera cualitativa, sin hacer una evaluación de la dosis respuesta. No obstante, cabe recordar que esta relación puede no ser evidente debido a que a diferentes dosis los efectos pueden ser distintos; por ejemplo, a ciertas dosis bajas pueden presentarse malformaciones $\mathrm{y}$, a dosis mayores, puede ocurrir muerte fetal o aborto, ${ }^{10}$ lo que impide detectar la asociación en estudios epidemiológicos de recién nacidos.

Una alternativa para la evaluación de la exposición es el empleo de marcadores biológicos. Los biomarcadores pueden ser alteraciones celulares bioquímicas o moleculares, que pueden medirse en tejidos, células y fluidos. Existen tres tipos de biomarcadores: de efecto, de exposición y de susceptibilidad. ${ }^{2}$ Empero, su empleo se dificulta ya que la mayoría de los estudios hacen una evaluación retrospectiva de la exposición, de tal manera que únicamente podrían emplearse los marcadores de exposición de gran persistencia en fluidos corporales, como las sustancias lipofílicas y algunos metales pesados ${ }^{27}$ que se depositan en grasa o hueso.

El periodo de exposición importante para que se produzcan los efectos teratogénicos durante el embarazo, también llamado ventana crítica de exposición, puede ser muy específico. El periodo de sensibilidad máxima difiere para cada órgano y depende, en su mayor parte, del periodo de organogénesis máxima. En la especie humana, el sistema nervioso presenta, junto con el corazón, la sensibilidad más temprana. ${ }^{28} \mathrm{El}$ periodo de mayor vulnerabilidad para que ocurran los DTN es entre los días 15 y 30, debido a que el cierre del tubo neural ocurre entre el día 23 y 26 para el neuroporo cefálico y el 26 y 30 para el neuroporo caudal. Así, a menos que la sustancia permanezca en los fluidos corporales, las exposiciones agudas durante los primeros quince días, entre la fecundación y la implantación, tendrían poco o ningún efecto sobre el producto. Por otro lado, al considerar las exposiciones paternas deberá tenerse en cuenta que el periodo de espermatogénesis toma de 63 a 74 días, por lo que se deberán considerar las exposiciones en los tres meses previos al embarazo. ${ }^{10}$

Para ilustrar las dificultades en la evaluación de exposición se presentará el caso de los plaguicidas. Es- 
tudios experimentales en animales apoyan la hipótesis de la causalidad relacionada con esta exposición. ${ }^{4}$ En varios trabajos se ha notificado un incremento en el riesgo de anencefalia y espina bífida en hijos de trabajadores (hombres) agrícolas;, ${ }^{29-33}$ la exposición a plaguicidas se estimó a través del título de la ocupación como trabajador agrícola. En otro estudio epidemiológico que se realizó recientemente, se informó acerca de un pequeño incremento en el riesgo de DTN en hijos de mujeres expuestas a plaguicidas, habiendo estimado la exposición a través de cuestionario y juicio de expertos. ${ }^{34}$ Uno de los problemas que se presentan en este tipo de investigaciones es el de poder identificar al agente causal; el supuesto de estos estudios es que el trabajo agrícola es un buen indicador de exposición a plaguicidas. ${ }^{33}$ Sin embargo, el trabajo agrícola se relaciona también con otros factores que podrían ser relevantes en la etiología de las malformaciones.

La ocupación del padre en tanto que trabajador agrícola se relaciona con situaciones como vivir cerca de áreas de cultivo, con la aplicación de agroquímicos en general, así como con algunos hábitos como el uso de leña para cocinar. Además de la exposición a agroquímicos, las madres residentes en áreas rurales podrían padecer algunas deficiencias nutricionales involucradas con los procesos enzimáticos relacionados con la migración celular necesaria para el cierre del tubo neural.

Por otro lado, en la interpretación de la asociación reportada con hipertermia durante el embarazo, infecciones y uso de salicilatos, debe considerarse que estos procesos pueden relacionarse con las malformaciones a través del agente infeccioso, el incremento en la temperatura corporal de la madre, o la interferencia con la nutrición materna. ${ }^{20} \mathrm{Si}$ bien se ha informado que los salicilatos, acetaminofén y análogos inducen anencefalia en ratas, ${ }^{35}$ en estudios en humanos es difícil diferenciar entre estas drogas y la enfermedad materna que determina su uso. ${ }^{11}$

\section{Factores de riesgo para DTN estudiados}

Entre los factores de riesgo estudiados, aunque no concluyentes, se encuentran tanto los genéticos como los ambientales. Se ha notificado una mayor incidencia de DTN y pérdida fetal entre sujetos homocigotos para distintos alelos del complejo mayor de histocompatibilidad. ${ }^{36}$ Por otra parte, se han descrito pocas alteraciones cromosómicas y genes autosómicos recesivos en los productos malformados, aunque los informes más recientes apuntan hacia una interacción entre mutaciones relacionadas con el metabolismo de los folatos y la deficiencia de los mismos en la dieta.
Como ya se ha dicho, probablemente los factores ambientales son los más estudiados. No obstante, esas evaluaciones han sido poco específicas: por ejemplo, se han reportado mayores riesgos para personal femenino de enfermería. ${ }^{37}$ Las exposiciones maternas más específicas que se han estudiado son las exposiciones a solventes, ${ }^{38}$ radiaciones ionizantes y gases anestésicos. Las ocupaciones paternas de mayor riesgo corresponden a los trabajadores agrícolas o jornale$\operatorname{ros}^{39,33} \mathrm{y}$, después, las exposiciones a solventes, ${ }^{29,40}$ plaguicidas ${ }_{,}^{29}$ radiaciones ionizantes ${ }^{41}$ mercurio y productos de limpieza, que frecuentemente incluyen solventes. ${ }^{42,29}$ Pocos son los estudios sobre exposiciones ambientales no ocupacionales, tales como la residencia cercana a sitios de almacenamiento de desechos peligrosos ${ }^{43}$ el incremento en la prevalencia de DTN en áreas de alto uso de agroquímicos ${ }^{44}$ y la contaminación del agua con nitratos ${ }^{45} \mathrm{y}$ trialometanos. ${ }^{46}$

Por otra parte, también se ha estudiado el efecto de algunos medicamentos como los salicilatos, ${ }^{47}$ las drogas antineoplásicas y los anticonvulsivantes. Sin embargo, las asociaciones han sido débiles, excepto para el valproato de sodio en cuyo caso parece existir mayor consistencia de asociación. ${ }^{48-50}$ Asimismo, se han encontrado ciertos herbicidas relacionados a DTN, en particular el ácido triclorofenoxiacético. ${ }^{44}$

Desde hace algún tiempo se le presta gran atención a los factores de la dieta, como las deficiencias de zinc, folatos y otras vitaminas. ${ }^{51,52}$ No obstante, las mayores evindencias se vinculan con la suplementación de ácido fólic ${ }^{53,54}$ en el periodo periconcepcional y una reducción del riesgo de recurrencias e incidencia de DTN. . $^{15,55-57}$

El efecto protector del ácido fólico ha llevado a los investigadores a buscar variantes de uno o más genes candidatos involucrados en el metabolismos del ácido fólico, defectos que podrían evitarse con la ingesta periconcepcional de ácido fólico. ${ }^{58,59}$ Los fibroblastos de fetos con DTN presentan, con mayor frecuencia, una mutación genética relacionada con termolabilidad de la enzima 5,10 metilentetrahidrofolato reductasa (MTHFR), ${ }^{58}$ sobre todo con espina bífida. ${ }^{60,61}$ También se ha reportado mayor frecuencia de homocigotos padres de estos niños. ${ }^{60}$

La enzima MTHFR cataliza la reducción del 5,10 metilentetrahidrofolato a 5 metiltetrahidrofolato, precursor de una cadena de reacciones de metilación de sustancias de interés biológico en la síntesis de DNA. En 1994 Goyette y colaboradores ${ }^{62}$ aislaron el cDNA para MTHFR, y Frosst y colaboradores (1995) ${ }^{63}$ aislaron una variación polimórfica en la posición 677 del nucleótido que involucra el cambio de una base $\mathrm{C}$ a T, resultando en una sustitución de valina (GTC) por 
alanina (GCC), lo que resulta en una enzima termolábil. Las personas con esta variante termolábil de MTHFR tienen una reducción del $50 \%$ en la actividad enzimática, elevada hemocisteina sérica, folato sérico por abajo de lo normal, y se sospecha que tienen mayor riesgo de padecer enfermedad cardiovascular. ${ }^{64}$ Se ha informado, asimismo, que la suplementación de ácido fólico puede bajar la homocisteína plasmática en personas con esta variante genética. ${ }^{64}$ Como los DTN se han asociado a anormalidades en la homocisteína ${ }^{65,66,}$ y la suplementación con ácido fólico disminuye la homocisteína sérica en adultos con la variante termolábil de MTHFR, es posible que uno de los mecanismos biológicos en el que se basa el efecto protector del ácido fólico, se relacione con esta variante.

En estudios epidemiológicos llevados a cabo recientemente se ha encontrado que el ser homocigoto para el polimorfismo 677C-T del gene de la MTHFR se asocia con un incremento en el riesgo de DTN, con razones de momios de 7.2 y de 2.7 para aquellos que tienen al menos un alelo $677 \mathrm{C}-\mathrm{T} .{ }^{58} \mathrm{La}$ frecuencia de homocigotos para 677C-T en la población control de algunos de estos estudios ha sido baja (entre 5 y $6 \%$ ). Sin embargo, en otro estudio que se efectuó en Inglaterra ${ }^{67}$ no se encontró diferencia en las frecuencias de las variantes de MTHFR, con una frecuencia en casos y controles bastante más elevada de la informada en estudios anteriores. Lo anterior lleva a pensar que pueden existir variaciones importantes entre poblaciones y que la selección de los grupos que se van a comparar es de suma importancia. Empero, es muy importante tomar en cuenta que el genotipo por sí mismo puede no ser el mayor determinante del resultado, sino el efecto de la interacción entre el genotipo y el estado de los folatos ${ }^{68} \mathrm{u}$ otras exposiciones ambientales. En un estudio realizado por Ou cy y colaboradores ${ }^{58}$ se observó que el genotipo de MTHFR influenció los niveles de homocisteina únicamente cuando los niveles de folatos estaban por debajo del valor de la media. Por otra parte, todavía es materia de discusión si en el mecanismo biológico de esta mutación es mediado por el genotipo de la madre, del feto, o por una combinación de ambos.

\section{Conclusiones}

Existen bases para sospechar que algunas exposiciones ambientales tienen efecto sobre el cierre del tubo neural. Sin embargo, para reducir las incertidumbres atribuidas a los métodos de investigación, en los próximos estudios en humanos deberán tomarse en cuenta las siguientes recomendaciones:
- Las comparaciones geográficas y temporales sólo podrán realizarse con sistemas de vigilancia completos que incluyan a todas las instituciones del sistema de salud, así como las malformaciones que llevan a la defunción, y aquellas compatibles con la vida. Además, estos sistemas de vigilancia deberán emplear criterios de clasificación comunes. En los informes se deberán indicar claramente los métodos de búsqueda y registro de casos, la edad gestacional considerada para los abortos y muertes fetales, y si estos se incluyen en el denominador para el cálculo de las prevalencias.

- Las comparaciones deben realizarse, preferentemente, para las malformaciones específicas y no para grandes grupos de malformaciones, evitando formar grupos heterogéneos en su posible etiología.

- En la evaluación de exposiciones ambientales es necesario: a) tomar en cuenta las exposiciones maternas y las paternas; b) considerar el periodo crítico de exposición, esto es, tres meses anteriores a la concepción en el caso del padre, y un mes alrededor de la concepción para la madre; c) evaluar cuantitativamente la exposición, en la medida de lo posible, evitando clasificar a los grupos sólo como expuestos y no expuestos. Por otra parte, es recomendable el empleo de marcadores biológicos de exposición siempre que sea posible, así como el uso de marcadores biológicos que permitan clasificar a la población en grupos con distinta susceptibilidad genética.

\section{Referencias}

1. Edmonds LD, Layde PM, James LM, Flynt JW, Erickson JD, O zkley GP. Congenital malformations surveillance:Two American systems. Int J Epidemiol 1981;10(3):247-252.

2. Lynberg MC, Khoury MJ. Interaction between Epidemiology and Laboratory Sciences in the Study of Birth Defects Risk Factor Surveillance in Metropolitan Atlanta. J Toxicol Environ Health 1993;40:435-444.

3. Shaw GM, Gold EB. Methodological considerations in the study of parental occupational exposures and congenital malformations in offsprings. Scand J W ork Environ Health 1998;14:344-355.

4. Sever LE, Lynberg MC, Edmonds LD. The impact of congenital malformations on public health. Teratology. 1993:48(6):547-549.

5. Mutchinick 0, O rozco E, Lisker R, Babinski V. Programa Mexicano de Registro y Vigilancia Epidemiológica de Malformaciones Congénitas Externas. Salud Publica Mex 1988;30:88-100.

6.0 Ishan AF,Teschke K, Baird PA. Paternal occupation and congenital anomalies in offspring. Am J Ind Med 1991;20:447-457.

7. Sever LE. Looking for causes of neural tube defects:W here does the environment fit in? Environ Health Perspect 1995;103 suppl 6:165-171. 
8.Trevathan E, Chávez GF, Sever LE. Epidemiology of congenital malformations of the central nervous system En:Anderson DW. N euroepidemiology: A tribute to Bruce Schoenberg. Boca Raton, Florida: CRC Press. 1991;218-237.

9. Busam KJ, Roberts DJ, Golden JA. C linical teratology counseling and consultation case report: two distinct anterior neural tube defects in a human fetus: Evidence for an intermitent pattern of neural tube closure.Teratology 1993;48:399-403.

10. Elwood JM, Little J, Elwood JH. Epidemiology and control of neural tube defects. 0 xford : 0 xford Medical Publications, 1992.

11. Kline J, Stein Z, Susser M. Conception to birth. Epidemiology of prenatal development. N ueva York: O xford U niversity Press, 1989.

12. Medical Research Council.Vitamin Study Research Group. Prevention of neural tube defects : Results of the Medical Research Council Vitamin Study. Lancet 1991;338:131-137.

13. Shaw GM, Schaffer D,Velie EM, Morland K, Harris JA. Periconceptional vitamin use, dietary folate, and the occurrence of neural tube deffects. Epidemiology 1995;6: 219-226.

14. Hemminki K, Mutanen P, Saloniemi I, Luoma K. Congenital malformatios and maternal occupation in Finland : Multivariate analysis. J Epidemiol Community Health 1981;35:5-10.

15. Shaw GW, Harris JA. N eural tube defects-W hy area rates high among populations of Mexican descent? Environ Health Perspect 1995;103suppl6:163-164.

16. Borman B, Cryer C. Fallacies of international and national comparisons of disease ocurrence in the epidemiology of neural tube defects. Teratology 1990;42:405-412.

17. Lekea V, Tzoumaka-Bakuola C, G olding J. Incidence of anencephalus and spina bifida in Greece. Teratology 1988;38:347-349.

18. Hook EB. Incidence and prevalence as measures of the frequency of birth defects. Am J Epidemiol 1982;116(5):743-747.

19. Secretaría de Salud. Manual para la Vigilancia Epidemiológica de los D efectos del Tubo N eural. México, D.F.: Dirección General de Epidemiología, SSA, 0 ctubre 1993.

20. Ruiz-Matuz C, Fernandez-Q uintanilla G , Luna-G uzmán P,Tapia-C onyer R. Panorama epidemiológico de los defectos del tubo neural en México. Gac Med Mex 1995;131: 485-489.

21. Holmes LB, D riscoll, SG , A tkins L. Etiologic heterogenecity of neuraltube deffects. N Eng J Med 1976;294:365-369.

22. Khoury MJ, Erickson JD, James LM. Etiologic heterogenecity of neural tube deffects: Clues from epidemiology. Am J Epidemiol 1982;115: 358-548.

23. Khoury MJ, Cordero JF, G reenberg F, James L M, Erickson JD. Population study of the VACETERL Association: Evidence for its etiologic heterogenecity. Pediatrics 1983;71:815-820.

24. Lammer EJ, Sever LE, 0 akley G P.Teratogen update:Valproic acid.Teratology 1987;35: 465-473.

25. Restrepo M, Muñoz N , D ay N E, Parra JE, Romero L, N euyen-Dinsh $X$. Prevalence of adverse reproductive outcomes in a population occupationally exposed to pesticides in Colombia. Scand JW ork Environ Health 1990;16:232-238.

26. García AM. 0 ccupational exposure to pesticides and congenital malformations :A review of mechanisms, methods, and results. Am J Ind Med 1998;33(3):232-240.

27. Schulte PA, Perera FP, ed. Molecular epidemiology: Principles and practices. San Diego :A cademic Press, 1993.

28. Picar J. Toxicologie du developpement. Bélgica: Universidad Católica de Lovaina, 1989.

29. Brender JD, Suárez L. Paternal occupation and anencephaly. Am J Epidemiol 1990;131(3):517-521.

30. Fedrick J.Anencephalus in the 0 xford record linkage study area. Develop Med Child N eurol 1976;18:643-656.

31. Balarajan R, MCD owall M. Congenital malformations and agricultural workers. Lancet 1983;1:112-113.
32. Golding J, Sladden T. C ongenital malformations and agricultural workers. Lancet 1983;1:1393.

33. K ristensen $P$, Irgens $L M$, Andersen A. Birth defects among offspring of N orwegian farmers, 1967-1991. Epidemiology 1997;8:537-544.

34. Shaw GM,Velie EM, Katz EA, Morland KB, Harris JA. Maternal occupational exposures and risk factors for neural tube defects. Am J Epidemiol 1996;143:S1.

35. W alkany J, Kalter H. Congenital malformations. N Eng J Med 1961; 265:1046-1052.

36. Lappe M: HLA homozygosity and neural-tube defects. Lancet 1979; 23:1342.

37. Mate TD, Mulinare J. Erickson JD. Case-control study of congenital defects and parenteral employment in health care. Am J Ind Med 1993; 124:11-23.

38. Holmberg PC. Central nervous-system defects in children born to mothers exposed to organic solvents during pregnancy. Lancet 1979 28:177-179.

39. Polednak AP, Janerich DT. U ses of available record systems in epidemiologic studies of reproductive toxicology. Am J Ind Med 1983:4:329-348. 40. Louik C. Mitchell AA 0 ccupational exposures and birth defects: Final performance report 1RO $10 \mathrm{H} 02598-01 \mathrm{Al}$. Cincinnati $(\mathrm{OH}): \mathrm{N}$ ational Institute for O ccupational Safety and Health, 1992.

41. Sever LE, Ilbert ES, Hessol N A, M Intyre J. A case-control study of congenital malformations and occupational exposure to low-level ionizing radiation. Am J Epidemiol 1988;127:226-242.

42. Blatter BM, Hermens R, Bakker M, Roeleveld N,Verbeek ALM, Zielhuis GA. Paternal occupational exposure around conception and spina bifida in offspring. Am J Ind Med 1997; 32:283-291.

43. G eschwind SA, Stolwijik JAJ, Bracken M, Fitzgerald E, Stark A, 0 Isen C et al. Risk of congenital malformations associated with proximity to hazardous waste sites. Am J Epidemiol 1992;135:1197-1207.

44. Field B, Kerr C. Herbicide use and incidence of neural-tube defects (letter). Lancet 1979;1:1341-1342.

45. Dorsch MM, Scragg RKR, McMichael AJ, Baghurst PA, Dyer KF. Congenital malformations and maternal drinking water supply in rural South Australia:A case-control study. Am J Epidemiol 1984;119:473-486.

46. Environmental Protection Agency (EPA). W ashington :International Life Sciences Institute Risk Science. A review of evidence on reproductive and developmental effects of disinfection by products in drinking water. N orth Carolina: Research Triangle Park N C 1993.

47. Joschko MA, D reosti IE, Tulsi RS. The teratogenic effects of salicylic acid on the developing nervous system in rats in vitro.Teratology 1993;48: 105-114.

48. Finnel RH, Bennet GD, Karras SV, Mohl VK. Common hierarchies of susceptibility to the induction of neural tube defects in mouse embryos by valproic acid and its 4-propyl4-pentenoic acid metabolite. Teratology 1988;38: 313-320.

49. 0 akeshott P, Hunt GM.Valproate and spina bifida. Br Med J 1989;298: $1300-1301$

50. Lindhout $D$, Schmidt $D$. In utero exposure to valproate and neural tube defects. Lancet 1986;14:1392-1393.

51. Kalter $\mathrm{H}, \mathrm{W}$ arkany J. Congenital malformations. Etiologic factors and their role in prevention. $N$ Eng J Med 1983;308:424-431.

52. Knox EG, Lancashire RJ. Epidemiology of congenital malformations. Londres: :U niversity of Birmingham. Her Majestys Stationary O ffice 1991. 53. Hearey ChD, H arris JA, U satin MS, Epstein D M, U ry HK, N eutra RR. Investigation of a cluster of anencephaly and spina bifida. Am J Epidemiol 1984;120(4):559-564.

54. Czeizel AE. Congenital abnormalities are preventable. Epidemiology 1995;6(3):205-206.

55. Medical Research Council.Vitamin Study Research Group. Prevention of neural tube defects: Results of the Medical Research Council Vitamin Study. Lancet 1991;338:131-137. 
56. Rosenberg $\mathrm{H}$. Folic acid and neural tube defects-time for action? N Engl J Med 1992;327:1875-1877.

57. Mulinare J, Erickson JD . Prevention of neural tube defects. Teratology 1997;56:17-18.

58. 0 u CY, Stevenson RE, BroawnVK, Schwartz CE. 5,10 Methylentetrahydrofolate reductasa genetic polymorphism as risk factor for neural tube defects. Am J Med G enet 1996; 63:610-614.

59. Tom KA, Eskes B. Folates and the fetus. EurJ O bstet Gynecol Reprod Biol1997;71:105-111.

60.Van der Put N M, Steegers-Theunissen RPM, Frosst P,Trijbels FJM. Mutated methylenetetrahydrofolate reductasa as a risk factor for spina bifida. Lancet 1995;46:1070-1071.

61.W hitehead AS, Gallagher P, Mills JL. A genetic defect in 5,10 methylenetetrahydrofolate reductase in neural tube defects. Q J Med 1995;88: 763-766.

62. Goyette P, Summer JS, Milos R et al. Human methyleneterahydrofolate reductase: Isolation of $C D N A$, mapping and mutation identification. $\mathrm{Na}$ ture Genet 1994;7:195-200.
63. Frosst $P, B l o m ~ H J$, Milos $R$ et al. A candidate genetic riskfactor for vascular disease: A common mutation in methylenetetrahydrofolate reductase. $N$ at Genet 1995;10:111-113.

64. Kang SS, Zhou J,W omng PW K, Kowalisyn J, Strokosh G. Intermediate homocysteinemia: A thermolabile variant of methylenetetrahydrofolate reductase. Am J Hum Genet 1988:43:414-421.

65. Steegers-Theunissen RP. Folate metabolism and neural tube defects:A review. Eur J O bstet Gynecol Reprod Biol 1995;61:39-48.

66. Mills Jl, McPartin JM, Kirke P. Homocysteine metabolism in pregnancies complicated by neural tube defects. Lancet 1995;345:149-151.

67. Papapetrou C, Lynch SA, Burn J, Edwars YH. Methylenetetrahydrofolate reductase and neural tube defects. Lancet 1996;348:358.

68. Jacques PF, Bostrom AG,W illiams RR. Relation between folate status, a common mutation in methylenetetrahydrofolate reductase, and plasma homocysteine concentrations. Circulation 1996;93:7-9. 